\title{
Brownian Motion of Spiral Waves Driven by Spatiotemporal Structured Noise
}

\author{
I. Sendiña-Nadal, ${ }^{1}, *$ S. Alonso, ${ }^{2}$ V. Pérez-Muñuzuri, ${ }^{1}$ M. Gómez-Gesteira, ${ }^{1}$ V. Pérez-Villar, ${ }^{1}$ \\ L. Ramírez-Piscina, ${ }^{3}$ J. Casademunt, ${ }^{4}$ J. M. Sancho, ${ }^{4}$ and F. Sagués ${ }^{2}$ \\ ${ }^{1}$ Group of Nonlinear Physics, Faculty of Physics, University of Santiago de Compostela, 15706 Santiago de Compostela, Spain \\ ${ }^{2}$ Departament de Química Física, Universitat de Barcelona, Avenida Diagonal 647, 08028 Barcelona, Spain \\ ${ }^{3}$ Departament de Física Aplicada, Universitat Politècnica de Catalunya, Avenida Dr. Gregorio Marañón 44, 08028 Barcelona, Spain \\ ${ }^{4}$ Departament d'Estructura i Constituents de la Matèria, Universitat de Barcelona, Avenida Diagonal 647, 08028 Barcelona, Spain
} (Received 10 November 1999)

\begin{abstract}
Spiral chemical waves subjected to a spatiotemporal random excitability are experimentally and numerically investigated in relation to the light-sensitive Belousov-Zhabotinsky reaction. Brownian motion is identified and characterized by an effective diffusion coefficient which shows a rather complex dependence on the time and length scales of the noise relative to those of the spiral. A kinematically based model is proposed whose results are in good qualitative agreement with experiments and numerics.
\end{abstract}

PACS numbers: 82.40.Bj, 05.45.Jn, 47.54. $+\mathrm{r}$

Spirals are generic structures in extended nonequilibrium systems. They are characteristic of many reactiondiffusion systems [1], the most paradigmatic experimental example being the Belousov-Zhabotinsky (BZ) reaction [2], and they have been observed in systems as complex as the heart muscle associated to cardiac fibrillation $[3,4]$. Spiral patterns appear also as elementary solutions of the complex Ginzburg-Landau (CGL) equation [5].

Beyond the standard description of spiral waves, their response to spatial and/or temporal forcing has been largely analyzed. Temporal resonance [6], drift of vortices due to parameter gradients [7,8] or external fields [9], and anchoring on localized defects [10] are among the most studied effects.

On the other hand, the influence of random heterogeneities on extended excitable systems has recently attracted much attention. Noise as an initiator of new spatial structures [11-13], or sustaining wave propagation in subexcitable media [14-16], is a subject of much theoretical and experimental interest. Complementarily, the role of superimposed disorder on preexisting spatiotemporal patterns has been examined, in relation to propagating pulses [17], to the dynamics of CGL spirals [18] and 3D structures [19].

In this paper, we study the effect of a spatiotemporal structured noise on the motion of a spiral wave for the photosensitive $\mathrm{BZ}$ reaction. In the absence of randomness, the spiral tip rotates quasirigidly around its core, with no net translational mobility. When the noise is switched on, Brownian diffusion of the spiral is observed, characterized by a nonmonotonous dependence on the parameters of the noise. These observations are confirmed numerically using a two-variable Oregonator model. The analysis is completed by proposing a simple theoretical model based on a kinematic approach [20], capturing the basic features observed in experiments.

Experiments were carried out in a Petri dish of $9 \mathrm{~cm}$ diameter. The catalyst, ruthenium bipyridil, is immobilized in a thin, $1 \mathrm{~mm}$ thick, film of silica gel, prepared as in [21].
A solution of catalyst-free $\mathrm{BZ}$ reaction (initial concentrations $0.18 M \mathrm{KBr}, 0.33 M$ malonic acid, $0.39 M \mathrm{NaBrO}_{3}$, and $0.50 \mathrm{M} \mathrm{H}_{2} \mathrm{SO}_{4}$ ) was poured onto the gel. The temperature was kept constant at $25 \pm 1{ }^{\circ} \mathrm{C}$. Spatiotemporal noise is introduced by projecting on to the Petri dish the desired patterned illumination, controlling the excitability of the system, by means of a video projector (SONY CPJ-D500). Experiments were captured in a video recorder through a charge-coupled device camera placed vertically. In order to enhance the contrast, an interference filter at $460 \mathrm{~nm}$ was placed before the camera lens. The images were digitized by an image-acquisition card (miroVideo DC30 plus) and analyzed on a PC.

The illumination field consisted of an array of square cells of size $\ell$ whose light intensity was varied on an eightbit gray scale between 0 and 255. In the illumination range used, the speed of planar autowaves decreases linearly with the light intensity [22]. With our random forcing, the light intensity in the cell $(i, j)$ is $I_{i j}(t)=I_{0}+\xi_{i j}(t)$, where the random term $\xi_{i j}(t)$ stands for a spatiotemporal Gaussian process, with a time independent zero mean average and a correlation function $\left\langle\xi_{i j}(t) \xi_{l m}\left(t^{\prime}\right)\right\rangle=\sigma^{2} \exp \left(-\left|t-t^{\prime}\right| /\right.$ $\tau) \delta_{i l} \delta_{j m}[23,24]$ (see Fig. 1). As a first step in examining the effects of spatiotemporal noise on spiral dynamics in what follows we report on a series of experiments conducted with fixed $\ell$ comparable to the size of the core. In each series we vary the correlation time $\tau$, keeping the noise dispersion $\sigma^{2}$ constant. For the sake of comparison, sets of experiments and numerical simulations were also performed with pure temporal noise (i.e., corresponding to the limit of infinite $\ell$ ). Experiments started with the generation of a free end in a square lattice of $2.5 \mathrm{~cm} \times$ $2.5 \mathrm{~cm}$ size by inhibiting one-half of a planar wave. After 5 min under uniform illumination, the medium was covered with a fully developed spiral of approximately nine wavelengths (wavelength $\lambda=0.27 \mathrm{~cm}$, period $T=45 \mathrm{~s}$, core size $d=0.11 \mathrm{~cm}$ ). An effective diffusion coefficient is calculated in terms of the tip mean square displacement by averaging over five trajectories. 

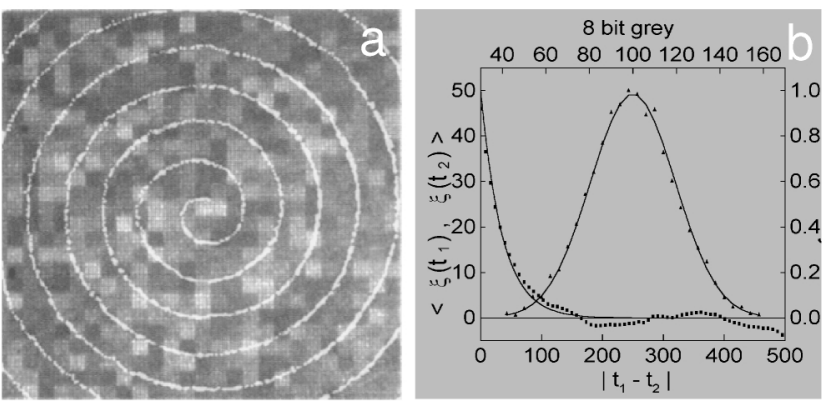

FIG. 1. Characterization of spatiotemporal noise employed in experiments. (a) Snapshot of a spiral wave (the background was subtracted to enhance the contrast) superposed to the illumination pattern with random intensity values at each cell $\left(\ell=0.9 \mathrm{~mm}, I_{0}=0.28 \mathrm{~mW} \mathrm{~cm}^{-2}\right)$. (b) Statistical properties of the fluctuating illumination: any cell in the array exhibits a Gaussian distribution centered in a relative gray value of 100 with a standard deviation of 40 . The temporal part of the autocorrelation function fits the exponential law given in the text for $\tau=35 \mathrm{~s}$.

Numerically, we used a two-variable Oregonator model adapted to our photosensitive medium [25],

$$
\begin{aligned}
& \partial_{t} u=\frac{1}{\varepsilon}\left(u-u^{2}-\left[f v+\phi_{i j}(t)\right] \frac{u-q}{u+q}\right)+D_{u} \nabla^{2} u, \\
& \partial_{t} v=(u-v)+D_{v} \nabla^{2} v,
\end{aligned}
$$

where $u$ (respectively, $v$ ) describes $\mathrm{HBrO}_{2}$ (respectively, catalyst) concentrations. $D_{u}$ and $D_{v}$ are diffusion coefficients, and $f, q$, and $\varepsilon$ are parameters related to the
BZ kinetics. $\phi_{i j}(t)$ represents the light-induced flow of $\mathrm{Br}^{-}$, directly proportional to the illumination $I_{i j}(t)$. Equations (1) were numerically integrated using a Euler method $[23,24]$ with a time step of $10^{-3}$ t.u. (time units) and a grid size of 0.16 s.u. (space units) in an array of $500 \times$ 500 points. Zero flux boundary conditions were considered. The tip coordinates of an initially prepared spiral ( $\lambda=9.6$ s.u., $T=1.69$ t.u., $d=3.2$ s.u.) were tracked following the point where the cross product of $u$ and $v$ gradients was maximal [26]. For each value of $\tau$, a number of roughly one hundred trajectories were simulated to reduce statistical dispersion.

The qualitative effect of $\tau$ on the Brownian motion of the spiral is illustrated in Fig. 2. Both experimentally 2(a)-2(c) and numerically 2(d)-2(f) it is found that its mobility largely depends on $\tau$. For values of $\tau$ smaller [Figs. 2(a) and 2(d)] and greater [Figs. 2(c) and 2(f)] than $\tau=T / 2 \pi, T$ being the rotation period, the trajectories are confined to a reduced part of the whole medium as enrolled filaments. Contrarily, for $\tau$ around $T / 2 \pi$ [Figs. 2(b) and 2(e)], trajectories span over a larger region, even reaching the boundary where the spiral finally dies out. Quantitative results are displayed in Fig. 3, together with experimental and numerical results corresponding to pure temporal noise. For finite $\ell$, the amplitude of the resonant peak increases and it appears at higher values of $\tau$.

In order to gain some theoretical insight, we use a kinematical approach appropriate to 2D spiral waves for weakly excitable media [20]. Although our experiments

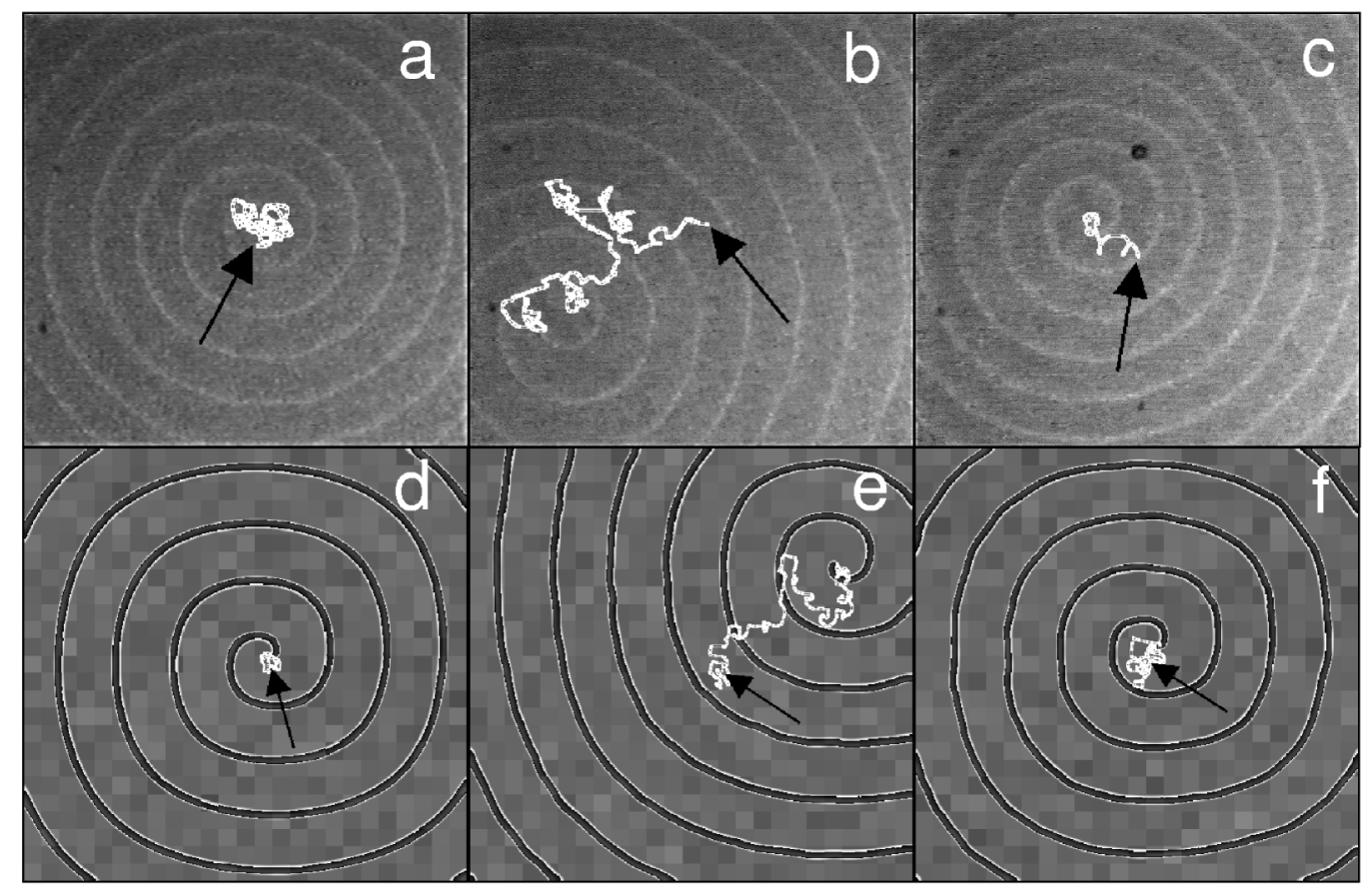

FIG. 2. Superimposition of the final spiral state and the whole trajectory of the tip for three different values of $\tau$ both experimentally (a)-(c) and numerically (d)-(f). In the experiment, the noise pattern was updated at $700 \mathrm{~ms}$ intervals and was interrupted during $10 \mathrm{~ms}$ every $35 \mathrm{~s}$ in order to capture a noise-free image of the wave activity. (a) $\tau=1 \mathrm{~s}$, (b) $\tau=35 \mathrm{~s}$, (c) $\tau=100 \mathrm{~s}$, (d) $\tau=1 \mathrm{t}$.u., (e) $\tau=20$ t.u., and (f) $\tau=100$ t.u. The arrow indicates the initial position of the tip. Experimentally, same parameters as in the caption of Fig. 1. Numerically, $\phi_{0}=0.01, \varepsilon=0.01, f=1.4, q=0.002, D_{u}=1, D_{v}=0.6, \sigma=0.0033$, and $\ell=3.2$ s.u. 

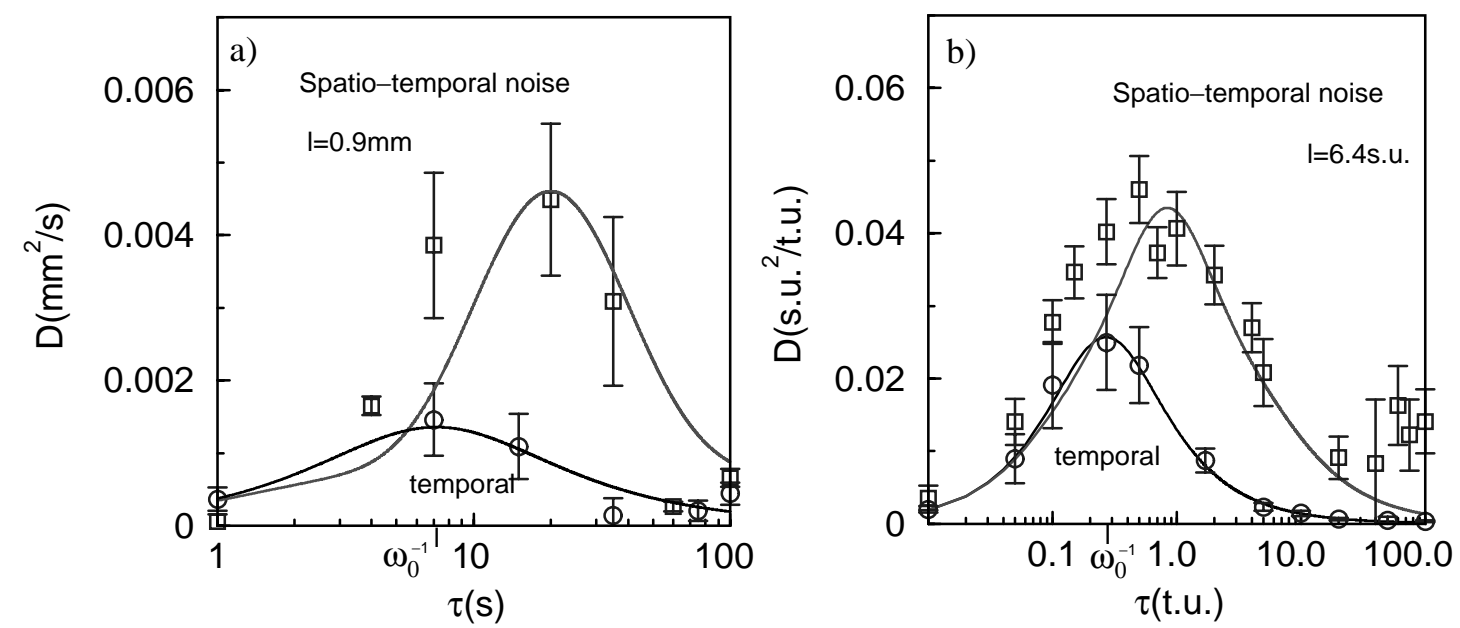

FIG. 3. Effective diffusion coefficient versus noise correlation time $\tau$. (a) Experimental results with parameters as in Fig. 1. Continuous lines correspond to the fits of our analytical results. Parameter values for the pure temporal case are $\sigma^{2}=0.00076$, $\omega_{0}=0.14$. Parameter values for the spatiotemporal case are $\sigma^{2}, \omega_{0}$ as before and $\frac{\bar{V}_{0}}{\omega_{0} \ell}=4, a=400$. (b) Results for the Oregonator model with parameters of Fig. 2, except $\ell=6.4$ s.u. Continuous lines correspond to the fits of our analytical results. Parameter values for the pure temporal case are $\sigma^{2}=0.38, \omega_{0}=3.7$. Parameter values for the spatiotemporal case are $\sigma^{2}, \omega_{0}$ as before and $\frac{\bar{V}_{0}}{\omega_{0} \ell}=1, a=0.7$. Error bars associated to the statistics of the experimental and numerical data are depicted.

fall beyond that limit, such a description enables us to capture, at least qualitatively, what we believe are the essential features of the effect of structured noise on well-formed spirals. For simplicity, we restrict the standard scheme to a situation with constant angular velocity $\omega_{0}$ and retain the equations for the tip position, together with the relaxation dynamics of the instantaneous curvature of the spiral at its free end,

$$
\begin{aligned}
\dot{x} & =-V_{0} \sin \omega_{0} t-\gamma\left(k_{c}-k_{0}\right) \cos \omega_{0} t, \\
\dot{y} & =V_{0} \cos \omega_{0} t-\gamma\left(k_{c}-k_{0}\right) \sin \omega_{0} t, \\
\dot{k}_{0} & =-\left(1 / \tau_{G}\right) \gamma\left(k_{0}-k_{c}\right) .
\end{aligned}
$$

Within the kinematic approach, it seems most natural to consider fluctuations of $V_{0}$, the tip velocity normal to the wave front, and/or the critical curvature $k_{c}$. Let us start with the simplest situation corresponding to $V_{0}(\mathbf{r}, t)=$ $\bar{V}_{0}+\xi(\mathbf{r}, t)$, but with no tangential velocity of the free end (no sprouting or contraction of the spiral curve): $k_{c}(t)=$ $k_{c}=k_{0}$. Within the spirit of the quasistatic approximation, implicit in the kinematic scheme above, the spatial structure of the noise will enter only through the successive positions visited by the tip. The problem is thus formally reduced to a zero dimensional one with a purely temporal noise defined by $\eta(t)=\xi(\mathbf{r}(t), t)$, where $\mathbf{r}(t)$ is the tip trajectory. For any arbitrary (stationary) temporal noise $\eta(t)$ added to $\bar{V}_{0}$, it can be shown that the diffusion coefficient $D$ of the tip position is given exactly in terms of the value of power spectrum of the noise $D=P_{\eta}\left(\omega_{0}\right)$ at the natural frequency $\omega_{0}$ [27]. The problem is thus reduced to the computation of such power spectrum for the effective temporal noise defined above. This cannot be accomplished exactly so we introduce a "quasideterministic" approximation by defining the effective noise as $\eta_{0}(t)=\xi\left(\mathbf{r}_{\mathbf{0}}(t), t\right)$, where $\mathbf{r}_{\mathbf{0}}(t)$ is the deterministic trajectory, which in our case corresponds to a circular motion with frequency $\omega_{0}$.

For the correlator corresponding to our experimental noise, the expression for the effective diffusion coefficient within the above mentioned approximation reads explicitly [27]

$$
\begin{aligned}
D=\frac{\sigma^{2}}{2} & {\left[\frac{\tau}{1+\omega_{0}^{2} \tau^{2}}+\frac{\bar{V}_{0}}{\omega_{0} \ell} \frac{\tau}{2}\right.} \\
& \left.\times\left(1-\frac{1+7 \omega_{0}^{2} \tau^{2}}{\left(1+\omega_{0}^{2} \tau^{2}\right)\left(1+4 \omega_{0}^{2} \tau^{2}\right)}\right)\right] .
\end{aligned}
$$

Let us first analyze the case of purely temporal noise $\left(\frac{\bar{V}_{0}}{\omega_{0} \ell} \ll 1\right.$, i.e., core radius much smaller than the noise correlation length). We obtain a resonantlike dependence of $D$ with $\tau=1 / \omega_{0}$, in full agreement with the experimental and numerical results. The physical interpretation of this behavior is as follows. At fixed noise dispersion $\sigma^{2}$, the effect of noise must disappear for vanishing $\tau$, since fast, bounded fluctuations will be averaged out by the system. On the other hand, for $\omega_{0} \tau \gg 1$, the spiral core loses mobility because the noise does not change appreciably during a rotation period of the tip. The effect of temporal noise is thus most effective when noise variations occur at the natural time scale of the spiral.

In the parameter region where $\frac{\bar{V}_{0}}{\omega_{0} \ell} \sim O(1), D$ is significantly enhanced, particularly near resonant conditions. This is again in accordance with the experimental and numerical observations. However, Eq. (3) predicts an artificial linear increase of $D$ at large $\tau$. The basic limitation of the quasideterministic ansatz which leads to Eq. (3) lies in the fact that the deterministic trajectory is closed. This introduces spurious effects at large correlation times of the 
noise since, for $\omega_{0} \tau \gg 1$, the values of the effective noise seen by the tip appear strongly correlated after every rotation period. In fact, the actual trajectory is not closing in itself and decorrelates the effective noise by exploring spatially uncorrelated regions.

Nevertheless, the model can be formally corrected for this effect by introducing an additional time scale $\tau^{*}$ which accounts for the above spatial decorrelation. Rather than attempting to go into a detailed discussion of the different mechanisms that may play a role in defining such a parameter, we concentrate on a crucial ingredient that has been missing so far: the role of fluctuations in $k_{c}$ [28]. It is worth mentioning at this point that numerical simulations of Eq. (2) (results not included in this Letter [27]) show a rather different behavior at values of $\tau \simeq T$, depending on whether noise is introduced only through $V_{0}$ or on both $V_{0}$ and $k_{c}$. Without noise on $k_{c}$, there is a tendency of the tip motion to attach its quasicircular motion to the boundaries between cells and propagate along them. Contrarily when noise is added also through $k_{c}$ this effect is avoided, the tip being confined most of the time inside the cells except for relatively fast transits between them. We recognize that this is nothing but a signature of a sort of pinning effect, as is indeed also evidenced by experiments and numerical treatment of the Oregonator model at large values of $\tau$. According to this mechanism, an heuristic argument [27] can be invoked which predicts an asymptotic decay of $D$ for large $\tau$ of the form $D \sim 1 / \tau$. This functional dependence can be incorporated into our scheme for $\frac{\bar{V}_{0}}{\omega_{0} \ell} \sim O(1)$, by defining $\tau^{*} \equiv a / \tau$ and further replacing $\tau$ by $\tau_{\text {eff }}\left(1 / \tau_{\text {eff }} \equiv 1 / \tau+1 / \tau^{*}\right)$ in Eq. (3).

Comparisons of this effective theory with the experimental and numerical results are shown in Fig. 3. The good agreement between the analytical prediction Eq. (3) [29] (with the appropriate $\tau_{\text {eff }}$ ) and both experiments and numerical integration of the Oregonator model, for a very large range of parameters, suggests that the model proposed, in spite of its apparent simplicity, does capture the basic physical mechanisms of the problem.

This work was partially supported by the Comision Interministerial de Ciencia y Tecnología (DGES), Consellería de Educación e Ordenación Universitaria (Xunta de Galicia), and Comissionat per a Universitats i Recerca (Generalitat de Catalunya) under Projects No. PB96-1001, No. PB96-0241, No. PB97-0540, No. XUGA20602B97, and No. 1997SGR00090.

*Corresponding author.

Email address: irene@fmmeteo.usc.es

[1] Chemical Waves and Patterns, edited by R. Kapral and K. Showalter (Kluwer Academic, Dordrecht, 1993).

[2] A. N. Zaikin and A. M. Zhabotinsky, Nature (London) 225, 535 (1970).
[3] J. M. Davidenko, A. M. Pertsov, R. Salomonz, W. Baxter, and J. Jalife, Nature (London) 335, 349 (1992).

[4] A. M. Pertsov, J. M. Davidenko, R. Salomonz, W. Baxter, and J. Jalife, Circ. Res. 72, 631 (1992).

[5] Y. Kuramoto, Chemical Oscillations, Waves and Turbulence (Springer, Tokyo, 1984).

[6] K. I. Agladze, V. A. Davydov, and A. S. Mikhailov, Sov. Phys. JETP Lett. 45, 767 (1987).

[7] M. Markus, Zh. Nagy-Ungvarai, and B. Hess, Science 257, 225 (1992).

[8] V. G. Fast and A. M. Pertsov, J. Cardiovasc. Electrophysiol. 3, 255 (1992).

[9] O. Steinbock, J. Schütze, and S. C. Müller, Phys. Rev. Lett. 68, 248 (1992).

[10] A. P. Muñuzuri, V. Pérez-Muñuzuri, and V. Pérez-Villar, Phys. Rev. E 58, R2689 (1998).

[11] J. Maselko and K. Showalter, Physica (Amsterdam) 49D, 21 (1991).

[12] J. Wang, S. Kàdàr, P. Jung, and K. Showalter, Phys. Rev. Lett. 82, 855 (1999).

[13] J. García-Ojalvo and L. Schimansky-Geier, Europhys. Lett. 47, 298 (1999).

[14] P. Jung and G. Mayer-Kress, Phys. Rev. Lett. 74, 2130 (1995).

[15] S. Kàdàr, J. Wang, and K. Showalter, Nature (London) 391, 770 (1998).

[16] H. Hempel, L. Schimansky-Geier, and J. García-Ojalvo, Phys. Rev. Lett. 82, 3713 (1999).

[17] I. Sendiña-Nadal, A.P. Muñuzuri, D. Vives, V. PérezMuñuzuri, J. Casademunt, L. Ramírez-Piscina, J. M. Sancho, and F. Sagués, Phys. Rev. Lett. 80, 5437 (1998).

[18] I. S. Aranson, H. Chaté, and L.-H. Tang, Phys. Rev. Lett. 80, 2646 (1998).

[19] V. Pérez-Muñuzuri, F. Sagués, and J.M. Sancho (to be published).

[20] A. S. Mikhailov, V. A. Davydov, and V. S. Zykov, Physica (Amsterdam) 70D, 1 (1994).

[21] T. Yamaguchi, L. Kuhnert, Zs. Nagy-Ungvaray, S. C. Müller, and B. Hess, J. Phys. Chem. 95, 5831 (1991).

[22] I. Sendiña-Nadal, M. Gómez-Gesteira, V. Pérez-Muñuzuri, V. Pérez-Villar, J. Armero, L. Ramírez-Piscina, J. Casademunt, F. Sagués, and J. M. Sancho, Phys. Rev. E 56, 6298 (1997).

[23] J. García-Ojalvo and J. M. Sancho, Noise in Spatially Extended Systems (Springer-Verlag, New-York, 1999).

[24] J. García-Ojalvo, J. M. Sancho, and L. Ramírez-Piscina, Phys. Lett. A 168, 35 (1992).

[25] H.-J. Krug, L. Pohlmann, and L. Kuhnert, J. Phys. Chem. 94, 4862 (1990).

[26] W. Jahnke, W. E. Skaggs, and A. T. Winfree, J. Phys. Chem. 93, 740 (1989).

[27] S. Alonso et al. (to be published).

[28] According to complementary simulations of the kinematic scheme and Oregonator model, fluctuations in $V_{0}$ and $k_{c}$ are assumed to be proportional.

[29] To circumvent somehow the limitations of the kinematic approach as applied here in relation to the real experimental parameters, we choose to consider the dimensionless quantity $\frac{\bar{V}_{0}}{\omega_{0} \ell}$ in Eq. (3) as a fitting parameter. 